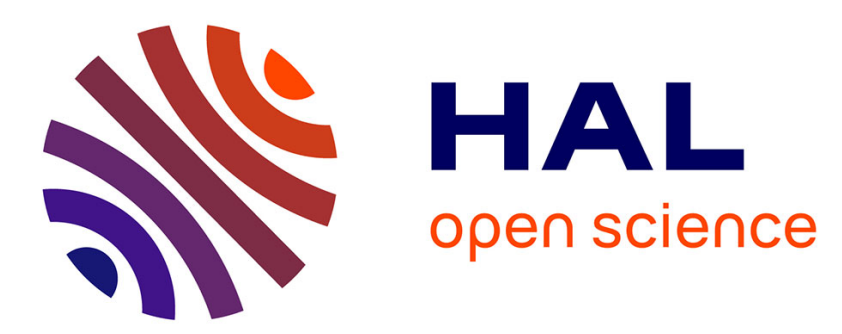

\title{
The Influence of Coupled Faradaic and Charging Currents on Impedance Spectroscopy
}

Shao-Ling Wu, Mark E. Orazem, Bernard Tribollet, Vincent Vivier

\section{To cite this version:}

Shao-Ling Wu, Mark E. Orazem, Bernard Tribollet, Vincent Vivier. The Influence of Coupled Faradaic and Charging Currents on Impedance Spectroscopy. Electrochimica Acta, 2014, 131, pp.3-12. 10.1016/j.electacta.2014.01.120 . hal-01019331

\section{HAL Id: hal-01019331 https://hal.sorbonne-universite.fr/hal-01019331}

Submitted on 12 Jan 2015

HAL is a multi-disciplinary open access archive for the deposit and dissemination of scientific research documents, whether they are published or not. The documents may come from teaching and research institutions in France or abroad, or from public or private research centers.
L'archive ouverte pluridisciplinaire HAL, est destinée au dépôt et à la diffusion de documents scientifiques de niveau recherche, publiés ou non, émanant des établissements d'enseignement et de recherche français ou étrangers, des laboratoires publics ou privés. 


\title{
The Influence of Coupled Faradaic and Charging Currents on Impedance Spectroscopy ${ }^{\text {th }}$
}

\author{
Shao-Ling $\mathrm{Wu}^{\mathrm{a}}$, Mark E. Orazem ${ }^{\mathrm{a}, *}$, Bernard Tribollet ${ }^{\mathrm{b}}$, Vincent Vivier ${ }^{\mathrm{b}}$ \\ ${ }^{a}$ Department of Chemical Engineering, University of Florida, Gainesville, FL, 32611, USA \\ ${ }^{b}$ LISE, UPR 15 du CNRS, Université P. et M. Curie, CP 133, 4 Place Jussieu, 75252 Paris cedex 05 France
}

\begin{abstract}
The coupling of faradaic and charging currents is shown, by numerical simulation, to result in impedance responses showing high-frequency frequency dispersion that resembles pseudo-ConstantPhase-Element (CPE) behavior. The results show that coupling of faradaic and charging currents should be considered when modeling the impedance response for systems influenced by mass transfer, even for large concentrations of supporting electrolyte.
\end{abstract}

Keywords: effective capacitance, frequency dispersion, CPE tungsten corrosion

\section{Introduction}

A controversy from the late 1960s over the correct method for developing deterministic models for impedance response has been largely neglected by the electrochemical community until it was raised in 2012 by Nisancioglu and Newman.[1] In electrochemical systems, the passage of current through an electrode can be attributed to faradaic reactions and to double-layer charging. As proposed by Sluyters, [2] the two processes are usually considered separately for simulating impedance response. The total current is subsequently obtained by adding the double-layer charging current to the faradaic current. This approach was criticized by Delahay and co-workers[3-5] because part of the flux of reacting species should, in principle, contribute to the charging of the interface as well as to the faradaic reaction. Nevertheless, the accepted procedure for model development has been to assume that the faradaic and charging currents are independent.[6] Indeed, the formalism in which faradaic and charging processes are considered to be uncoupled is documented in standard textbooks on impedance spectroscopy. $[7,8]$

The opposing viewpoints are presented schematically in Figure 1. With a-priori separation (APS) of charging and faradaic currents, the flux of reacting species contributes only to the faradaic reaction, as shown in Figure 1(a), and the charging current has contribution only from inert species. This corresponds to the currently accepted procedure for model development. The schematic representation of the case with no a-priori separation (NAPS) of charging and faradaic currents is presented in Figure 1(b). The reacting species are seen to contribute to both the faradaic reaction

\footnotetext{
This document published as Electrochimica Acta, 131 (2014), 3-12.

* Corresponding author

Email address: meo@che.uf1.edu (Mark E. Orazem)
} 


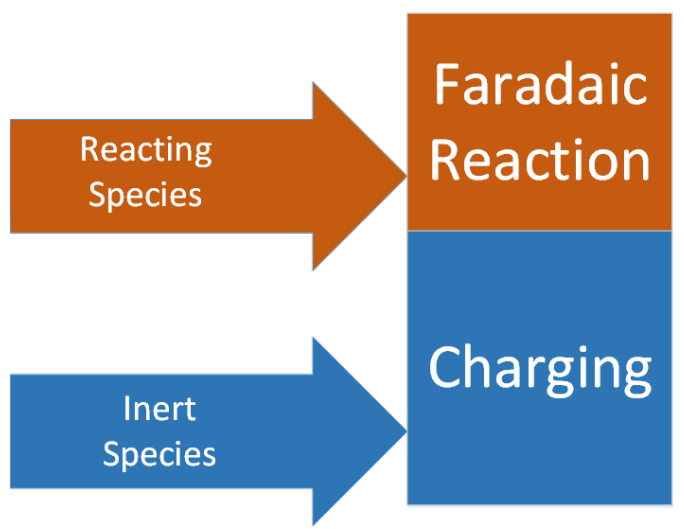

(a) APS

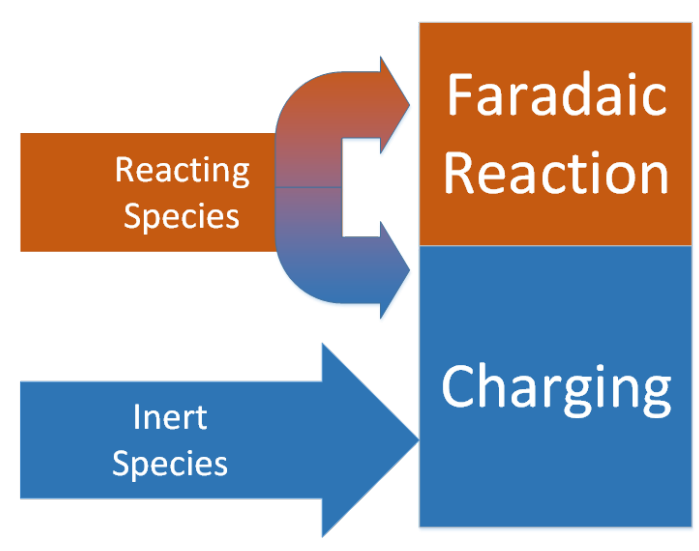

(b) NAPS

Figure 1: Schematic representation illustrating the contribution of the reacting species to the charging of the electrodeelectrolyte interface corresponding to: a) the case with a-priori separation (APS); and b) the case with no a-priori separation (NAPS).

and, along with the inert species, to the charging current. The representation shown in Figure 1(b) is consistent with the arguments presented by Delahay. [3-5]

Relaxation of the assumption that faradaic and charging currents are independent requires coupling an explicit model of the double layer to the convective diffusion equations for each ionic species. Nisancioglu and Newman[1] provided an appropriate mathematical formalism that provides the foundation for the present work. Nisancioglu and Newman suggest that the coupling of faradaic and charging currents must be considered in general, but that the effect is not significant for wellsupported electrolytes for which the reacting species contributes little to the interfacial charge. The object of this work is to use numerical simulations to explore the influence that the coupling of faradaic and charging currents has on the impedance response.

\section{Model Development}

A two-dimensional impedance model was developed to study the effect of nonuniform mass transfer, rotating disk electrode geometry, and the coupling of charging and faradaic currents on the impedance response.[9]

\subsection{Mass Transport in Dilute Solutions}

Conservation of species is expressed as

$$
\frac{\partial c_{\mathrm{i}}}{\partial t}=-\nabla \cdot \mathbf{N}_{\mathrm{i}}+R_{\mathrm{i}}
$$

where the flux is given by

$$
\mathbf{N}_{\mathrm{i}}=-D_{\mathrm{i}}\left(z_{\mathrm{i}} c_{\mathrm{i}} \frac{\mathrm{F}}{\mathrm{R} T} \nabla \Phi+\nabla c_{\mathrm{i}}\right)+c_{\mathrm{i}} \mathbf{v}
$$

$c_{\mathrm{i}}$ is the concentration of species $\mathrm{i}, z_{\mathrm{i}}$ is the charge number, $D_{\mathrm{i}}$ is the diffusivity, $\mathbf{v}$ is the massaveraged velocity associated with the rotating disk, and $R_{\mathrm{i}}$ represents the production of species i 
by homogeneous reactions. In the absence of homogeneous reactions and under assumption that the diffusion coefficients are uniform,

$$
\frac{\partial c_{\mathrm{i}}}{\partial t}+\mathbf{v} \cdot \nabla c_{\mathrm{i}}=z_{\mathrm{i}} D_{\mathrm{i}} \frac{\mathrm{F}}{\mathrm{R} T} \nabla \cdot\left(c_{\mathrm{i}} \nabla \Phi\right)+D_{\mathrm{i}} \nabla^{2} c_{\mathrm{i}}
$$

For a system with $n$ species, $n$ expressions in the form of equation (3) are required; whereas the conservation of charge implies that

$$
\nabla \cdot \mathbf{i}=-\nabla \cdot\left(\mathrm{F} \sum_{\mathrm{i}} D_{\mathrm{i}} z_{\mathrm{i}}\left(z_{\mathrm{i}} c_{\mathrm{i}} \frac{\mathrm{F}}{\mathrm{R} T} \nabla \Phi+\nabla c_{\mathrm{i}}\right)\right)=0
$$

Equations (3) and (4) constitute a set of nonlinear equations.

In the frequency domain, the concentrations of each species are described by

$$
c_{\mathrm{i}}=\bar{c}_{\mathrm{i}}+\operatorname{Re}\left\{\widetilde{c}_{\mathrm{i}} \exp (\mathrm{j} \omega t)\right\}
$$

where the bar notation represents the steady-state component, and the tilde notation represents the oscillating component which depends only on position. Similar definitions are applied for all variables. The mass and charge conservation equations become

$$
\mathrm{j} \omega \widetilde{c}_{\mathrm{i}}+\mathbf{v} \cdot \nabla \widetilde{c}_{\mathrm{i}}=D_{\mathrm{i}} \nabla \cdot\left(\nabla \widetilde{c}_{\mathrm{i}}+\frac{z_{\mathrm{i}} \mathrm{F}}{\mathrm{R} T}\left(\bar{c}_{\mathrm{i}} \nabla \widetilde{\Phi}+\widetilde{c}_{\mathrm{i}} \nabla \bar{\Phi}\right)\right)
$$

and

$$
\nabla \cdot\left(\sum_{\mathrm{i}} z_{\mathrm{i}} D_{\mathrm{i}}\left(\nabla \widetilde{c}_{\mathrm{i}}+\frac{z_{i} \mathrm{~F}}{\mathrm{R} T}\left(\bar{c}_{\mathrm{i}} \nabla \widetilde{\Phi}+\widetilde{c}_{\mathrm{i}} \nabla \bar{\Phi}\right)\right)\right)=0
$$

respectively, where the higher order terms such as $\widetilde{c_{\mathrm{i}}} \nabla \widetilde{\Phi}$ were neglected. At the electrode surface, the flux of each species may be expressed as

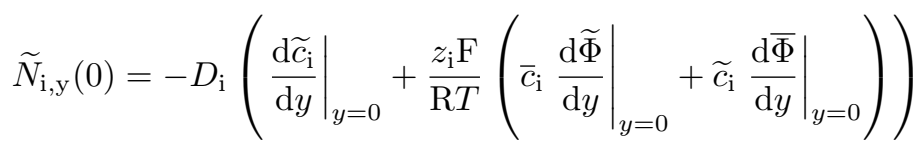

The correlation between the flux and the current oscillations at electrode boundary is discussed in two cases where the faradaic current and the double-layer charging current are considered with and without a priori separation of faradaic and charging currents.

\subsection{No a Priori Separation of Faradaic and Charging Currents (NAPS)}

Under the assumption that the charge density on the metal surface $q_{\mathrm{m}}$ is dependent on the interfacial potential $V$ and on the concentration $c_{\mathrm{i}}(0)$ of each species i, located outside the diffuse region of charge, the variation of the surface charge density is given by

$$
\mathrm{d} q_{\mathrm{m}}=\left(\frac{\partial q_{\mathrm{m}}}{\partial V}\right)_{c_{\mathrm{i}}(0)} \mathrm{d} V+\sum_{\mathrm{i}}\left(\frac{\partial q_{\mathrm{m}}}{\partial c_{\mathrm{i}}(0)}\right)_{V, c_{\mathrm{j} \neq(\mathrm{i})(0)}} \mathrm{d} c_{\mathrm{i}}(0)
$$

Equation (9) is written in terms of $n+1$ parameters which are treated as properties of the interface. These can be expressed as

$$
C_{0}=\left(\frac{\partial q_{\mathrm{m}}}{\partial V}\right)_{c_{\mathrm{i}}(0)}
$$


where $C_{0}$ is the usual differential capacitance, and

$$
C_{\mathrm{i}}=\left(\frac{\partial q_{\mathrm{m}}}{\partial c_{\mathrm{i}}(0)}\right)_{V, c_{\mathrm{j} \neq(\mathrm{i})(0)}}
$$

which can be expressed for species $\mathrm{i}=1, \ldots, n$. Both terms may be be obtained from detailed models of the diffuse double layer.

The current at the electrode surface may be expressed as

$$
\widetilde{i}=\mathrm{j} \omega \widetilde{q}_{\mathrm{m}}+\widetilde{i}_{\mathrm{F}}
$$

where the oscillations of the surface charge density and the faradaic current density are approximated by Taylor series expansions about their steady values as

$$
\widetilde{q}_{\mathrm{m}}=\left(\frac{\partial \bar{q}_{\mathrm{m}}}{\partial V}\right)_{c_{\mathrm{i}}(0)} \widetilde{V}+\sum_{\mathrm{i}}\left(\frac{\partial \bar{q}_{\mathrm{m}}}{\partial c_{\mathrm{i}}(0)}\right)_{V, c_{\mathrm{j} \neq(\mathrm{i})(0)}} \widetilde{c}_{\mathrm{i}}(0)
$$

and

$$
\widetilde{i}_{\mathrm{F}}=\left(\frac{\partial \bar{i}_{\mathrm{F}}}{\partial V}\right)_{c_{\mathrm{i}}(0)} \tilde{V}+\sum_{\mathrm{i}}\left(\frac{\partial \bar{i}_{\mathrm{F}}}{\partial c_{\mathrm{i}}(0)}\right)_{V, c_{\mathrm{j} \neq(\mathrm{i})(0)}} \widetilde{c}_{\mathrm{i}}(0)
$$

respectively. The surface flux can be expressed as

$$
\widetilde{N}_{\mathrm{i}, \mathrm{y}}(0)=-\frac{\partial \Gamma_{\mathrm{i}}}{\partial c_{\mathrm{i}}(0)} \frac{\partial c_{\mathrm{i}}(0)}{\partial q_{\mathrm{m}}} \mathrm{j} \omega \widetilde{q}_{\mathrm{m}}-\frac{s_{\mathrm{i}}}{n \mathrm{~F}} \widetilde{i}_{\mathrm{F}}
$$

where $s_{\mathrm{i}}$ is the stoichiometric coefficient for the reaction and $\Gamma_{\mathrm{i}}$ is the surface concentration of species i. Equations (12) and (15) were applied as the boundary conditions to evaluate the impedance response without a priori assumption of the separation of faradaic and charging currents.

\subsection{Double-Layer Model}

In the present study, the Gouy-Chapman-Stern model was used to describe the electrical behavior in the diffuse double layer. Under the assumption that ion-specific adsorption could be neglected, values for $C_{0}$ and $C_{\mathrm{i}}$, equations (10) and (11), respectively, were obtained as functions of potential and the concentration of individual ionic species at the outer limit of the diffuse part of the double layer. The structure of the electrical double layer is illustrated in Figure 2. The classical Gouy-Chapman theory shows an exponential decay of potential in a diffuse region of charge extending from the electrode surface. Stern's modification to the Gouy-Chapman model assumes a compact layer between the electrode and the diffuse layer. The outer limit of the compact layer is also referred to as the outer Helmholtz plane (OHP) which is the locus of centers of mobile species in their position of closest approach to the electrode surface. When there is specific adsorption on electrode surface, the locus of centers of adsorbed species is taken to be the inner Helmholtz plane (IHP).

As the thickness of the double layer is very thin, it is often considered to be a part of the electrode-electrolyte interface. The interfacial region as a whole obeys electrical neutrality, i.e., the excess surface charge density at electrode must be balanced by the surface charges at the IHP and in the diffuse part of the double layer such that

$$
q_{\mathrm{m}}+q_{\mathrm{ihp}}+q_{\mathrm{d}}=0
$$




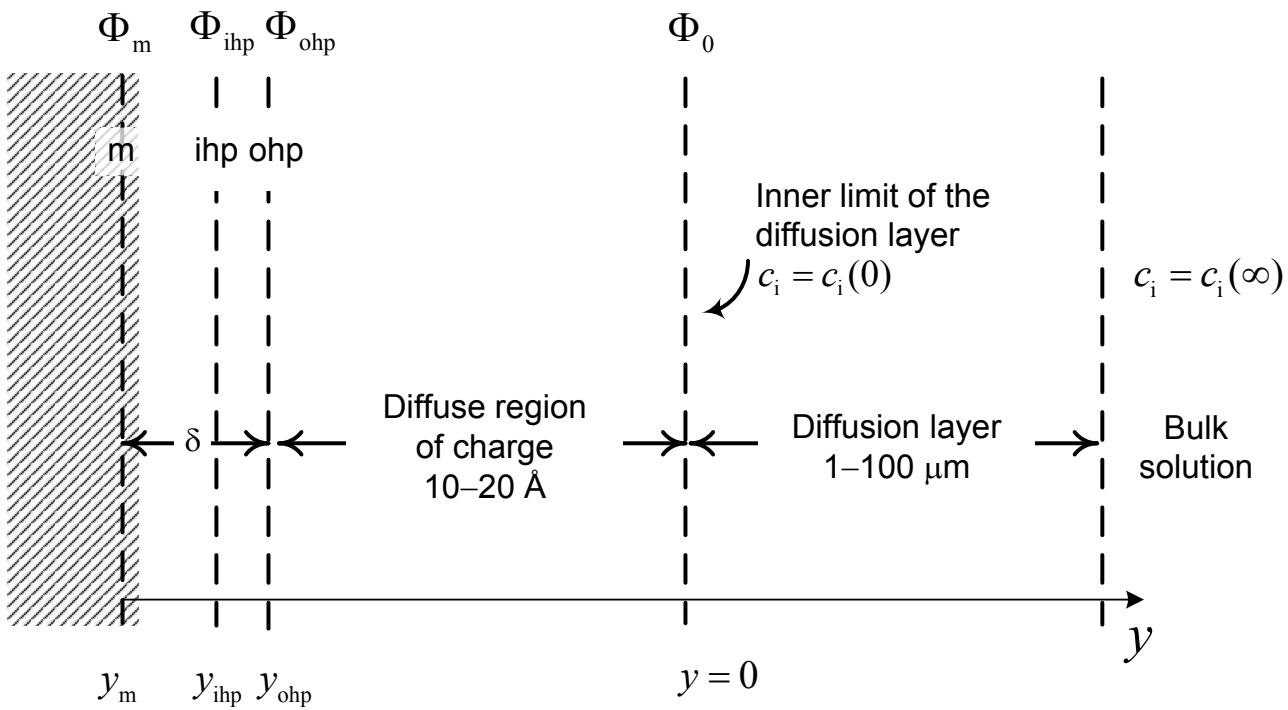

Figure 2: Schematic representation of the electrical double layer structure following a Stern-Gouy-Chapman model. The sketch is not drawn to scale.

The surface charge density is related to the surface excess concentration of charged species by

$$
q_{\mathrm{m}}=-\left(q_{\mathrm{ihp}}+q_{\mathrm{d}}\right)=-\mathrm{F} \sum_{\mathrm{i}} z_{\mathrm{i}} \Gamma_{\mathrm{i}}
$$

The mean electrostatic potentials at the metal surface, the IHP, and the OHP are denoted by $\Phi_{\mathrm{m}}$, $\Phi_{\mathrm{ihp}}$, and $\Phi_{\mathrm{ohp}}$, respectively. The surface concentrations $c_{\mathrm{i}}(0)$ and potential $\Phi_{0}$ used in the rate expressions are usually evaluated at the outer limit of the diffuse layer, or the inner limit of the diffusion layer.

Beyond the OHP, the total charge in the diffuse part of the double layer is not electrically neutral. The ionic concentrations in the diffuse layer were assumed to have a Boltzmann distribution, i.e.,

$$
c_{\mathrm{i}}=c_{\mathrm{i}}(\infty) \exp \left(-\frac{z_{\mathrm{i}} \mathrm{F} \Phi}{\mathrm{R} T}\right)
$$

Poisson's equation provides the relationship between the concentrations and potential as

$$
\frac{\mathrm{d}^{2} \Phi}{\mathrm{d} y^{2}}=-\frac{\mathrm{F}}{\epsilon_{\mathrm{d}} \epsilon_{0}} \sum_{\mathrm{i}} z_{\mathrm{i}} c_{\mathrm{i}}=-\frac{\mathrm{F}}{\epsilon_{\mathrm{d}} \epsilon_{0}} \sum_{\mathrm{i}} z_{\mathrm{i}} c_{\mathrm{i}}(\infty) \exp \left(-\frac{z_{\mathrm{i}} \mathrm{F} \Phi}{\mathrm{R} T}\right)
$$

where $y$ is the distance from the electrode, $\epsilon_{\mathrm{d}}$ is the dielectric constant in the diffuse layer, and $\epsilon_{0}$ is the permittivity of vacuum $\left(\epsilon_{0}=8.8542 \times 10^{-14} \mathrm{~F} / \mathrm{cm}\right)$. Integration of the Poisson equation gives the relation of potential gradient to the surface charge density in the diffuse layer as

$$
\left.\frac{\mathrm{d} \Phi}{\mathrm{d} y}\right|_{y_{\mathrm{ohp}}}=\frac{q_{\mathrm{d}}}{\epsilon_{\mathrm{d}} \epsilon_{0}}
$$


where $y_{\text {ohp }}$ is located at the inner limit of the diffuse part of the double layer, as indicated in Figure 2. At equilibrium, the potential approaches zero far from the electrode surface, and Poisson's equation can then be solved by applying the boundary conditions to yield the charge density in the diffuse layer, i.e.,

$$
q_{\mathrm{d}}=\mp\left\{2 \mathrm{R} T \epsilon_{\mathrm{d}} \epsilon_{0} \sum_{i} c_{\mathrm{i}}(\infty)\left[\exp \left(\frac{-z_{\mathrm{i}} \mathrm{F} \Phi_{\mathrm{ohp}}}{\mathrm{R} T}\right)-1\right]\right\}^{1 / 2}
$$

The upper sign is used if the potential is positive and, conversely, the lower sign is used if the potential is negative.

In the absence of ion-specific adsorption on the IHP, i.e., $q_{\mathrm{ihp}}=0$, the surface concentration is related to the individual charge density within the diffuse part of the double layer by

$$
\Gamma_{\mathrm{i}}=\frac{q_{\mathrm{d}, \mathrm{i}}}{z_{\mathrm{i}} \mathrm{F}}
$$

Following the convention adopted by Gibbs (see for example, Chapter 7 in reference [10]) the surface charge associated with a given species i within the diffuse part of the double layer can be expressed as

$$
q_{\mathrm{d}, \mathrm{i}}=z_{\mathrm{i}} \mathrm{F} \int_{y_{\mathrm{ohp}}}^{\infty}\left(c_{\mathrm{i}}-c_{\mathrm{i}}(\infty)\right) \mathrm{d} y
$$

where $c_{\mathrm{i}}$ is given by equation (18). Integration over $y$ is accomplished by use of the definition of electric field, i.e.,

$$
E=-\frac{\mathrm{d} \Phi}{\mathrm{d} y}
$$

where $E$ is obtained as a function of $\Phi$ from equations (20) and (21). The charge density associated with individual species is therefore obtained by an integral over the potential drop in the diffuse region as

$$
q_{\mathrm{d}, \mathrm{i}}=\mp \int_{0}^{\Phi_{\mathrm{ohp}}} \frac{z_{\mathrm{i}} \mathrm{F} c_{\mathrm{i}}(\infty)\left[\exp \left(\frac{-z_{\mathrm{i}} \mathrm{F} \Phi}{\mathrm{R} T}\right)-1\right]}{\left\{\frac{2 \mathrm{R} T}{\epsilon_{\mathrm{d}} \epsilon_{0}} \sum_{\mathrm{k}} c_{\mathrm{k}}(\infty)\left[\exp \left(\frac{-z_{\mathrm{k}} \mathrm{F} \Phi}{\mathrm{R} T}\right)-1\right]\right\}^{1 / 2}} \mathrm{~d} \Phi
$$

The above expressions for charge densities were derived by assuming a true equilibrium of the system in which the ionic concentrations at the outer limit of the diffuse layer are the same as the bulk values, i.e., $c_{\mathrm{i}}(0)=c_{\mathrm{i}}(\infty)$, and the potential at the outer limit of the diffuse layer is equal to that of a reference electrode placed at infinity; thus, for a reference electrode of the same kind as the working electrode, $\Phi_{0}=0$.

When the net current flowing to the electrode is not equal to zero, the system is not at equilibrium, i.e., $\Phi_{0} \neq 0$. Equations (21) and (25) become

$$
q_{\mathrm{d}}=\mp\left\{2 \mathrm{R} T \epsilon_{\mathrm{d}} \epsilon_{0} \sum_{\mathrm{i}} c_{\mathrm{i}}(0)\left[\exp \left(\frac{-z_{\mathrm{i}} \mathrm{F}\left(\Phi_{\mathrm{ohp}}-\Phi_{0}\right)}{\mathrm{R} T}\right)-1\right]\right\}^{1 / 2}
$$


and

$$
q_{\mathrm{d}, \mathrm{i}}=\mp \int_{0}^{\Phi_{\mathrm{ohp}}} \frac{z_{\mathrm{i}} \mathrm{F} c_{\mathrm{i}}(0)\left[\exp \left(\frac{-z_{\mathrm{i}} \mathrm{F} \Phi}{\mathrm{R} T}\right)-1\right]}{\left\{\frac{2 \mathrm{R} T}{\epsilon_{\mathrm{d}} \epsilon_{0}} \sum_{\mathrm{k}} c_{\mathrm{k}}(0)\left[\exp \left(\frac{-z_{\mathrm{k}} \mathrm{F} \Phi}{\mathrm{R} T}\right)-1\right]\right\}^{1 / 2}} \mathrm{~d} \Phi
$$

respectively. The charge densities in the diffuse layer are now related to the concentrations and potential at the outer limit of the diffuse layer, which can be obtained by solving the mass and charge conservation equations outside the diffuse region of charge.

The evaluation of the surface charge density in equation (21) or (26) requires additional information in the electrical double layer. Gauss's law relates the surface charge density to the electric field within the OHP by

$$
q_{\mathrm{m}}=-q_{\mathrm{d}}=\frac{\epsilon \epsilon_{0}}{\delta}\left(\Phi_{\mathrm{m}}-\Phi_{\mathrm{ohp}}\right)
$$

where $\epsilon$ is the dielectric constant between the metal surface and the OHP and $\delta$ is the distance between the metal surface and the OHP, as shown in Figure 2. Equation (28) can be used as a second equation to solve for $q_{\mathrm{m}}$ and $\Phi_{\mathrm{ohp}}$ in the electrical double layer.

\subsection{With a Priori Separation of Faradaic and Charging Currents (APS)}

The conventional approach is to consider the double-layer charging and the faradaic currents to be separable quantities. The total current density is expressed by

$$
i=i_{\mathrm{C}}+i_{\mathrm{F}}=C_{0} \frac{\mathrm{d} V}{\mathrm{~d} t}+i_{\mathrm{F}}
$$

or, in frequency domain,

$$
\widetilde{i}=\mathrm{j} \omega C_{0} \widetilde{V}+\widetilde{i}_{\mathrm{F}}=\mathrm{j} \omega\left(\frac{\partial q_{\mathrm{m}}}{\partial V}\right)_{c_{\mathrm{i}}(0)} \widetilde{V}+\widetilde{i}_{\mathrm{F}}
$$

The contribution of mass flux in charging the double layer is neglected when the faradaic current and the charging current are considered separately. Thus,

$$
\widetilde{N}_{\mathrm{i}, \mathrm{y}}(0)=-\frac{s_{\mathrm{i}}}{n \mathrm{~F}} \widetilde{i}_{\mathrm{F}}
$$

Comparison of equation (31) with equation (15) shows that the first term in equation (15) is neglected. Under the assumption that there is a priori separation of faradaic and charging currents, equations (30) and (31) are used as the boundary conditions to evaluate the transient responses of potential and concentrations.

\section{Numerical Simulation}

Numerical methods were used to solve the sets of equations corresponding to the steady-state distributions of concentration and the model for the diffuse double layer. These provided the parameters presented in equations (10) and (11) that were needed for the numerical solution of the coupled convective diffusion equations in frequency domain. 


\subsection{Steady-State Calculations}

Equations (3) and (4) were solved under the assumption of a steady state using the finiteelement package COMSOL Multiphysics ${ }^{\circledR}$ with the Nernst-Planck module in a $2 \mathrm{D}$ axial symmetric coordinate system. The domain size was identical to that used for the impedance calculations presented in section 3.3. The values for the concentrations at the inner limit of the diffusion layer were used as input for the double-layer model described in section 3.2, and, as the problem is nonlinear due to the explicit treatment of the migration term, the steady-state values of potential and concentration were used for the impedance calculations described in section 3.3.

\subsection{Double-Layer Properties}

The nonlinear equations relating to the charge and potential distributions in the electrical double layer were solved by using the Newton-Raphson method. For nonequilibrium systems, a local equilibrium was assumed in which the concentrations and potential at the outer limit of the diffuse layer were given by the steady-state solution of the convective diffusion equations. The radially dependent surface concentrations and potential gave a radial distribution of charge over the electrode surface. The surface-averaged value of charge was obtained from the surface-averaged values of concentration and potential. The charge in the diffuse layer associated with individual species was obtained by subsequent numerical integration of equation (25) or (27), using the MATLAB integration function, the adaptive Gauss-Kronrod quadrature method. The thickness of the compact layer between the metal surface and the OHP was assumed to be $3 \AA$. The dielectric constant in the compact region within the OHP was approximately 6 , according to Bockris[11] for a fully oriented water layer next to the electrode surface. The dielectric constant in the diffuse layer was assumed to be 78 , which is the value of water at room temperature.

\subsection{Impedance Calculations}

The equations were solved for a rotating disk electrode by using the finite-element package COMSOL Multiphysics ${ }^{\circledR}$ with the Nernst-Planck module in a 2D axial symmetric coordinate system. The domain size was 2,000 times larger than the disk radius in order to meet the assumption that the counterelectrode was located infinitely far from the electrode surface. The meshed domain used to calculate the coupled solution for potential and concentrations is shown in Figure 3. A coarse mesh was used for the domain distant from the electrode to reduce physical memory usage and calculation time. A finer mesh was applied in the region that is 20 times larger than the disk radius to capture the variation of potential in the vicinity of the electrode. Since the concentration of ionic species varies only in a small distance above the electrode surface, a much finer mesh was constructed in the region that is ten times larger than the characteristic thickness of the diffusion layer

$$
\delta_{\mathrm{N}}=\left(\frac{3 D_{\mathrm{i}}}{a \nu}\right)^{1 / 3}\left(\frac{\nu}{\Omega}\right)^{1 / 2}
$$

where $a=0.51023$ is the coefficient for the first term in the velocity expansion for the rotating disk, $\nu$ is the kinematic viscosity, and $\Omega$ is the disk rotation speed.[10] This approach captured the concentration variation at the electrode surface.

Steady-state solutions were used in the impedance model in the form of lookup tables from which appropriate values could be obtained by interpolation. The use of the same meshed domain in the steady-state and the impedance models reduced the error from interpolation between nodal

points. The distributions of the double-layer capacitance and other thermodynamic parameters at 


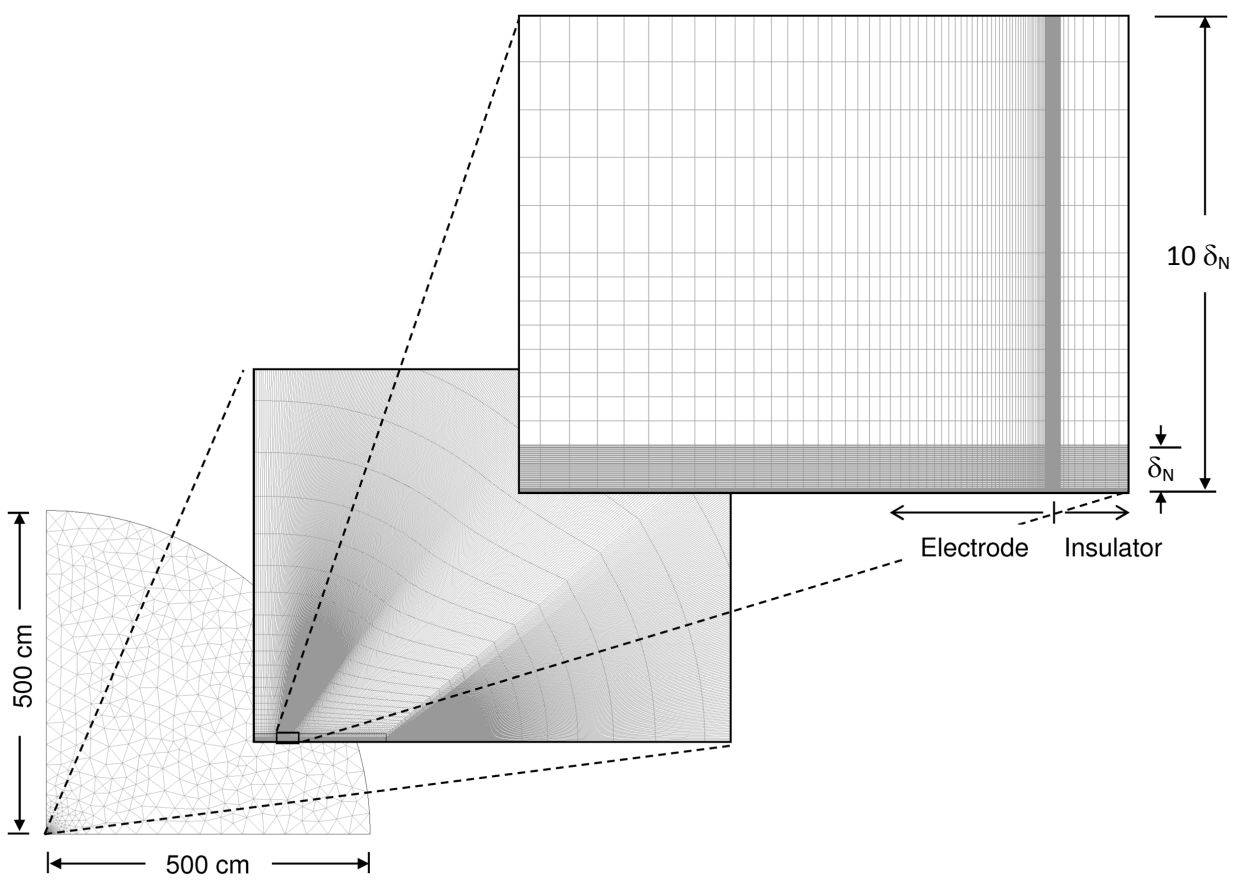

Figure 3: Representation of the different mesh scales used for the finite-element calculations. 


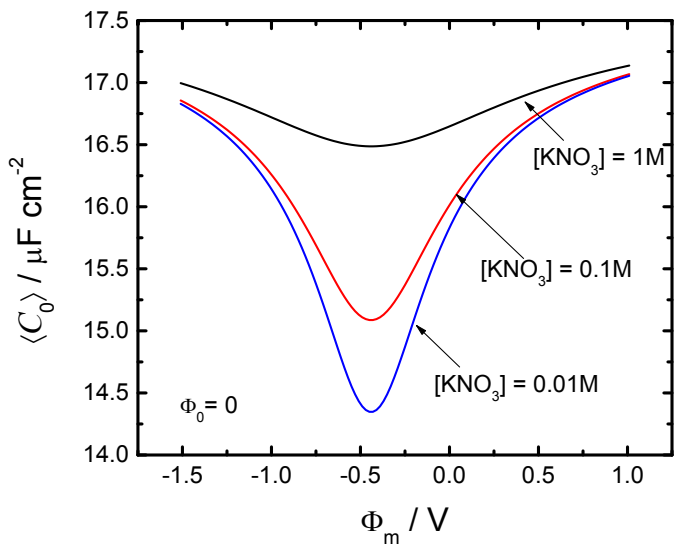

(a)

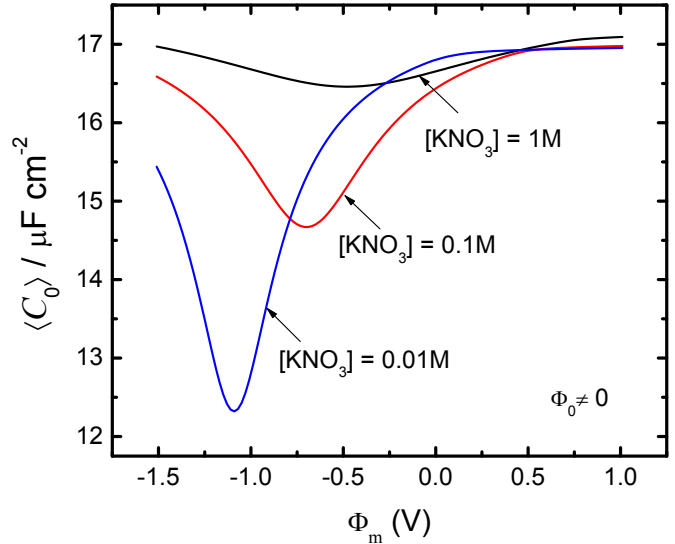

(b)

Figure 4: Calculated capacitance $C_{0}$ for $0.1 \mathrm{M} \mathrm{AgNO}_{3}$ as a function of potential for a silver electrode in solution containing $0.1 \mathrm{M} \mathrm{AgNO}_{3}$ and different concentrations of supporting electrolyte when the system is: a) at equilibrium and b) not at equilibrium.

electrode boundary were applied in the impedance model to evaluate the impedance response for the NAPS case. For the APS case, a surface-averaged double-layer capacitance

$$
\left\langle C_{0}\right\rangle=\frac{1}{\pi r_{0}^{2}} \int_{0}^{r_{0}} C_{0}(r) r \mathrm{~d} r
$$

was used to determine the charging current that did not include the contribution of mass transfer in the diffuse region of charge.

The velocity used in the expression for convective diffusion consisted of an interpolation formula that followed the three-term velocity expansion for radial and axial velocity components appropriate for the velocity boundary layer near the disk electrode surface and the two-term expansion appropriate for the region far from the disk surface (see Cochran[12]). The interpolation formula provided excellent accuracy near the electrode surface and showed good agreement with numerical solutions of the Navier-Stokes equations throughout the domain.

\section{Results}

The results of the thermodynamic simulations are presented to show the dependence of these quantities on potential and radial position. The impedance response employs the notation for global, local, local ohmic, and local interfacial impedances presented by Huang et al. [13-15] The results are explained in terms of an effective capacitance derived from equation (9).

\subsection{Thermodynamic Interfacial Properties}

For electrolytic solution containing symmetric electrolyte, the capacitance-potential curves are symmetric over $\Phi_{\mathrm{pzc}}$ when the system is at equilibrium as shown in Figure 4(a). The potential of zero charge for the silver electrode was assumed to be $\Phi_{\mathrm{pzc}}=-0.44 \mathrm{~V}$.[11] When the system is not 


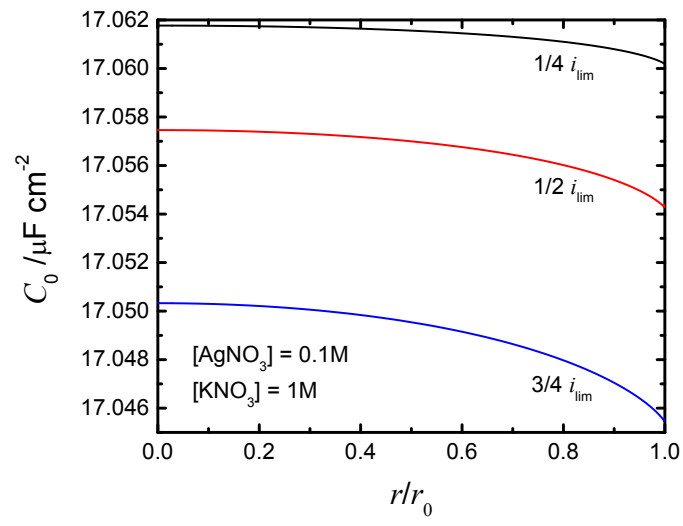

(a)

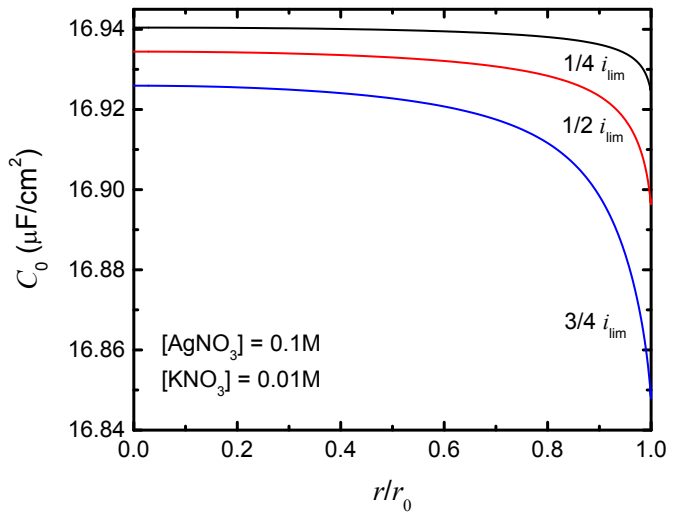

(b)

Figure 5: Calculated capacitance $C_{0}$ with fraction of mass-transfer-limiting current as a parameter for $0.1 \mathrm{M} \mathrm{AgNO}_{3}$ as a function of radial position for deposition of silver in an electrolyte containing $0.1 \mathrm{M} \mathrm{AgNO}_{3}$ and different concentrations of supporting electrolyte: a) $1 \mathrm{M} \mathrm{KNO}_{3}$; b) $0.01 \mathrm{M} \mathrm{KNO}_{3}$.

at equilibrium (e.g., Figure 4(b)), the minimum value of the double-layer capacitance is observed at a potential negative to $\Phi_{\mathrm{pzc}}=-0.44 \mathrm{~V}$. The double-layer capacitance shows larger dependency on electrode potential when the solution concentration is smaller, indicating the increasing potential gradient in the interfacial region with the decreasing solution conductivity.

The corresponding distribution of capacitance is presented in Figures 5(a) and 5(b) for an electrolyte containing $0.1 \mathrm{M} \mathrm{AgNO}_{3}$ and $1 \mathrm{M} \mathrm{KNO}_{3}$ or $0.01 \mathrm{M} \mathrm{KNO}_{3}$, respectively. Although the overall variations are not large, the reduced level of supporting electrolyte shown in Figure 5 (b) increases the interfacial potential and leads to a more nonuniform distribution of double-layer capacitance.

The surface averaged value $\left\langle\partial \Gamma_{\mathrm{i}} / \partial c_{\mathrm{i}}(0)\right\rangle$ which appears in equation (15) is presented in Figure 6(a) for an electrolyte containing $0.1 \mathrm{M} \mathrm{AgNO}_{3}$ and $1 \mathrm{M} \mathrm{KNO}_{3}$. The variation of the surface charge density with the concentration of each species at constant potential can be expressed by

$$
\left(\frac{\partial q_{\mathrm{m}}}{\partial c_{\mathrm{i}}(0)}\right)_{V}= \pm \frac{\exp \left(\frac{-z_{\mathrm{i}} \mathrm{F}\left(\Phi_{\mathrm{ohp}}-\Phi_{0}\right)}{\mathrm{R} T}\right)-1}{\left\{\frac{2}{\mathrm{R} T \epsilon_{\mathrm{d}} \epsilon_{0}} \sum_{\mathrm{i}} c_{\mathrm{i}}(0)\left[\exp \left(\frac{-z_{\mathrm{i}} \mathrm{F}\left(\Phi_{\mathrm{ohp}}-\Phi_{0}\right)}{\mathrm{R} T}\right)-1\right]\right\}^{1 / 2}}
$$

The upper sign is used when the potential across the diffuse part of the double layer is larger than the potential of zero charge, i.e., $\Phi_{\mathrm{ohp}}-\Phi_{0}>\Phi_{\mathrm{pzc}}$, and, conversely, the lower sign is used when $\Phi_{\mathrm{ohp}}-\Phi_{0}<\Phi_{\mathrm{pzc}}$. From equation (34), the variation of the surface charge density with ionic concentrations at a fixed potential is only dependent on the charge of each species. The resulting values for an electrolyte containing $0.1 \mathrm{M} \mathrm{AgNO}_{3}$ in a $1 \mathrm{M} \mathrm{KNO}_{3}$ are presented in Figure 6(b). The peak associated with $\partial q_{\mathrm{m}} / \partial c_{\mathrm{NO}_{3}^{-}}(0)$ occurs at an electrode potential at which the deposition of silver is no longer limited by the mass transfer of silver ion.

The radial distributions of $C_{\mathrm{i}}=\partial q_{\mathrm{m}} / \partial c_{\mathrm{i}}(0)$ are presented in Figure 7 with fraction of the masstransfer-limited current density as a parameter for an electrolyte containing $0.1 \mathrm{M} \mathrm{AgNO}_{3}$ in a $1 \mathrm{M}$ 


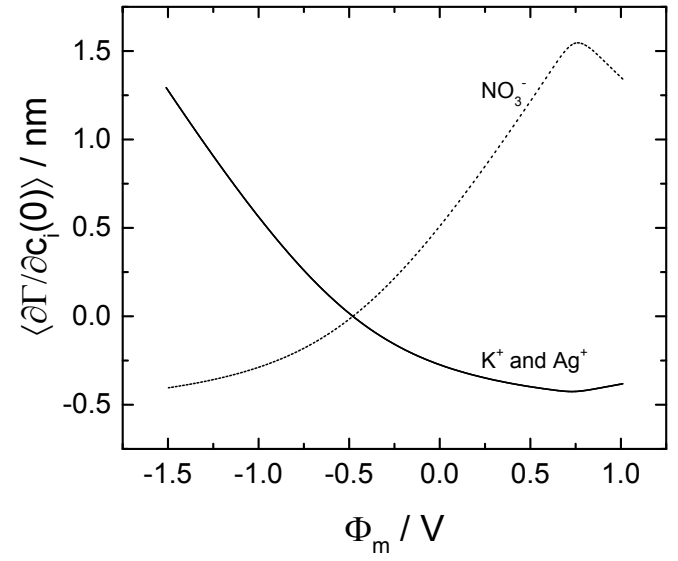

(a)

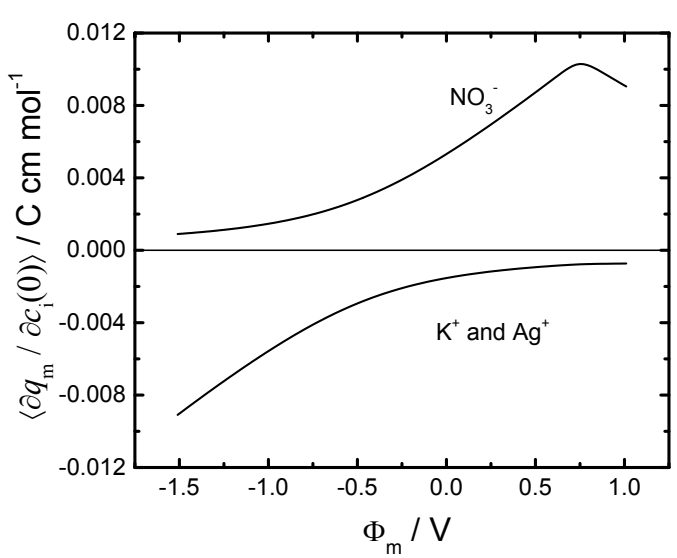

(b)

Figure 6: Calculated surface averaged quantity for $0.1 \mathrm{M} \mathrm{AgNO}_{3}$ in a $1 \mathrm{M} \mathrm{KNO}_{3}$ electrolyte: a) $\left\langle\partial \Gamma_{\mathrm{i}} / \partial c_{\mathrm{i}}(0)\right\rangle$; b) $\left\langle C_{\mathrm{i}}=\partial q_{\mathrm{m}} / \partial c_{\mathrm{i}}(0)\right\rangle$.

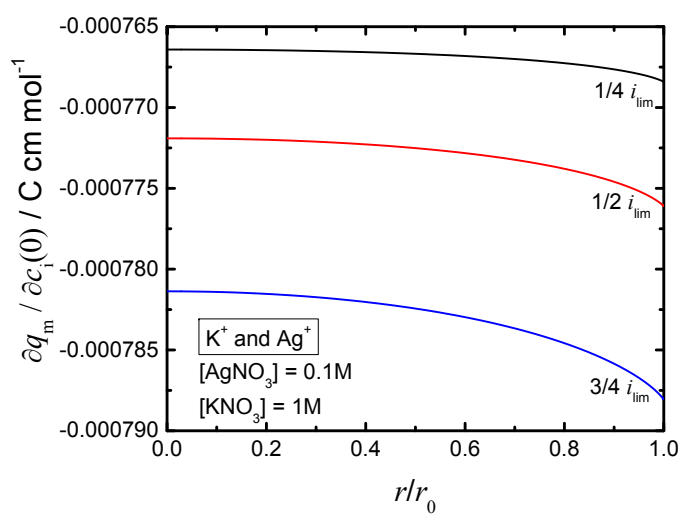

(a)

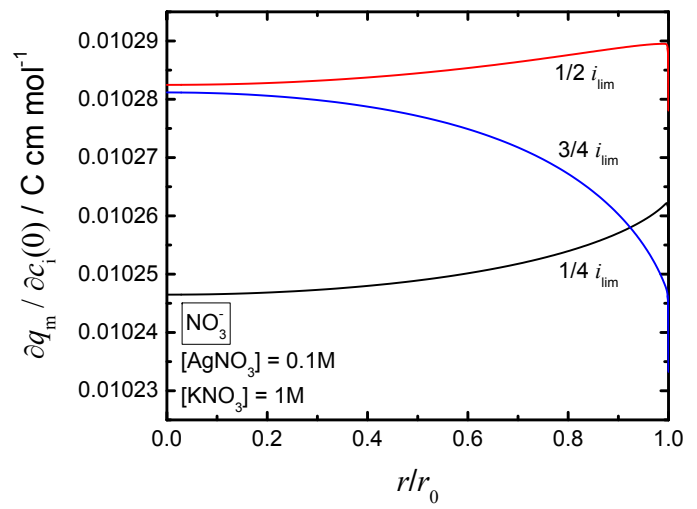

(b)

Figure 7: Calculated radial distribution of the quantity $C_{\mathrm{i}}=\partial q_{\mathrm{m}} / \partial c_{\mathrm{i}}$ with fraction of the mass-transfer-limited current density as a parameter for $0.1 \mathrm{M} \mathrm{AgNO}_{3}$ in a $1 \mathrm{M} \mathrm{KNO}_{3}$ electrolyte: a) $\mathrm{K}^{+}$and $\mathrm{Ag}^{+}$; and b) $\mathrm{NO}_{3}^{-}$. 


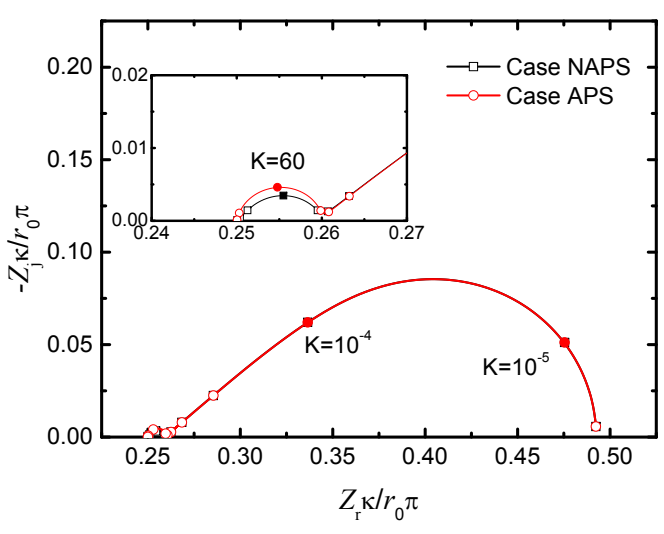

(a)

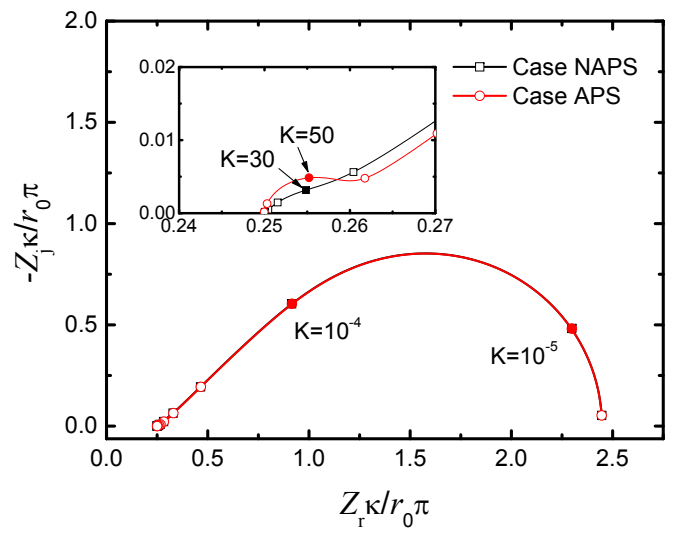

(b)

Figure 8: Calculated impedance response in Nyquist format for the case with a-priori separation (APS) and no a-priori separation (NAPS): a) $0.1 \mathrm{M} \mathrm{AgNO}_{3}$ in a $1 \mathrm{M} \mathrm{KNO}_{3}$ electrolyte; and b) $0.01 \mathrm{M} \mathrm{AgNO}_{3}$ in a $1 \mathrm{M} \mathrm{KNO}_{3}$ electrolyte.

$\mathrm{KNO}_{3}$ electrolyte. The results for $\mathrm{K}^{+}$and $\mathrm{Ag}^{+}$are presented in Figure $7(\mathrm{a})$ and the results for $\mathrm{NO}_{3}^{-}$are presented in Figure $7(\mathrm{~b})$. The quantity $C_{\mathrm{i}}=\partial q_{\mathrm{m}} / \partial c_{\mathrm{i}}$ is shown to be a weak function of radial position.

\subsection{Global Impedance}

The global and local impedance responses for the deposition of silver were calculated with different treatments of the boundary conditions (cases APS and case NAPS) and for different concentrations of supporting electrolyte. The calculated normalized global impedances calculated under assumption of APS and NAPS are presented in Figures 8(a) and 8(b) for electrolytes containing $0.1 \mathrm{M} \mathrm{AgNO}_{3}$ and $1 \mathrm{M} \mathrm{KNO}_{3}$ and $0.01 \mathrm{M} \mathrm{AgNO}_{3}$ and $1 \mathrm{M} \mathrm{KNO}_{3}$, respectively, where

$$
K=\frac{\omega\left\langle C_{0}\right\rangle r_{0}}{\kappa}
$$

represents a dimensionless frequency. The difference between a priori and no-a-priori separation of Faradaic and double-layer charging currents is observed at high frequencies where the faradaic and charging processes are equally important. At high-frequencies, a depressed semicircle is seen for both the APS and the NAPS cases. The degree of depression, however, is greater for the NAPS case.

The distinction between the APS and NAPS models can be seen more clearly in Figure 9, where the logarithm of the absolute value of the imaginary part of the global impedance is presented as a function of the logarithm of frequency. As discussed by Orazem et al. [16], the slope of the curves presented in Figure 9, presented in Figure 10 with fraction of the mass-transfer-limited current density as a parameter, can be related to the exponent of a constant phase element.

The depressed semicircle obtained for the APS case is very similar to that obtained by Huang et al. [15] for a secondary current distribution in which mass transfer effects were ignored. The slope at high frequency has a mild frequency dependence and approaches a value of $\alpha=0.9$ for the APS results presented in both Figures 10(a) and 10(b). Huang et al. [15] reported similar values 


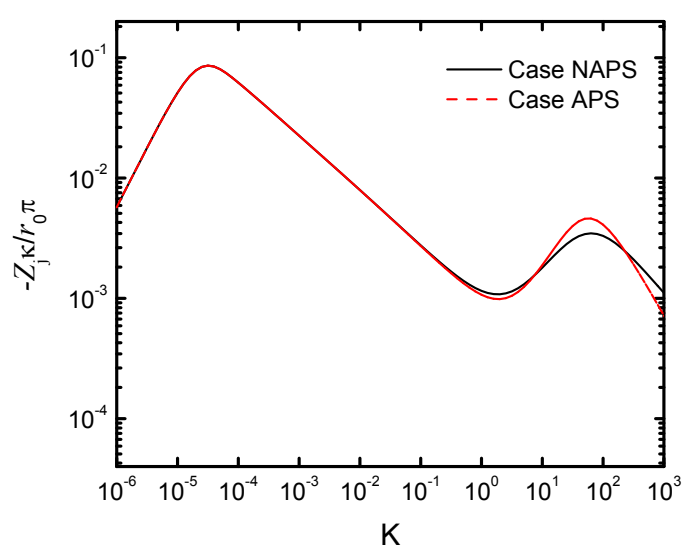

(a)

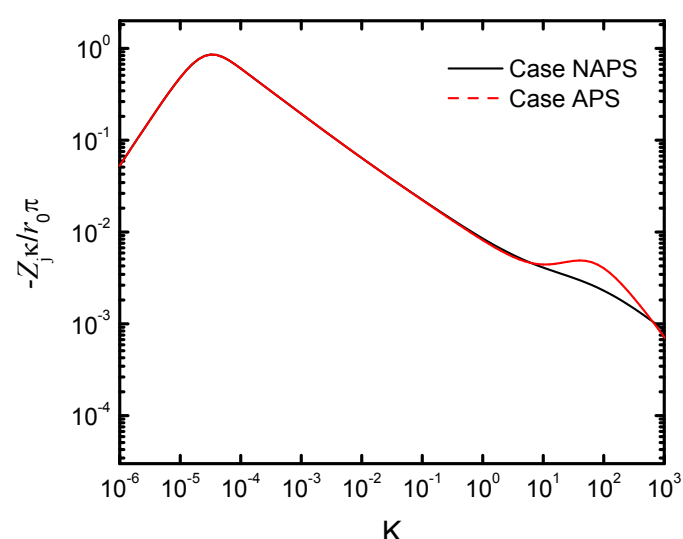

(b)

Figure 9: Calculated imaginary impedance response as a function of dimensionless frequency for the case with $a$ priori separation (APS) and no a-priori separation (NAPS): a) $0.1 \mathrm{M} \mathrm{AgNO}_{3}$ in a $1 \mathrm{M} \mathrm{KNO}_{3}$ electrolyte; and b) $0.01 \mathrm{M} \mathrm{AgNO}_{3}$ in a $1 \mathrm{M} \mathrm{KNO}_{3}$ electrolyte.

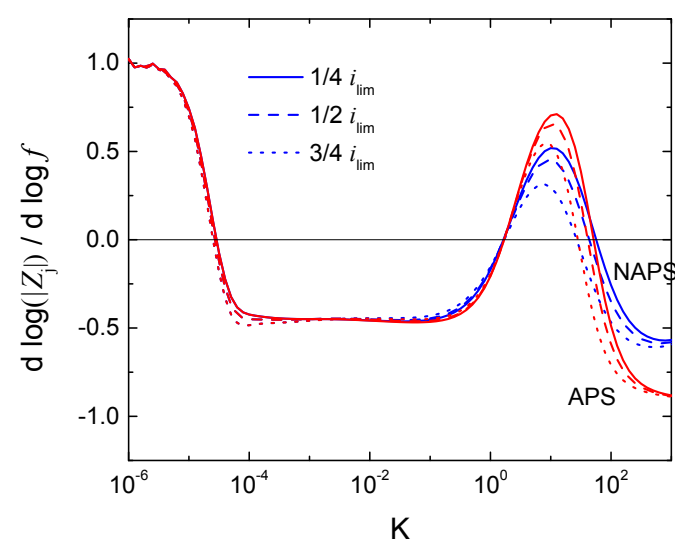

(a)

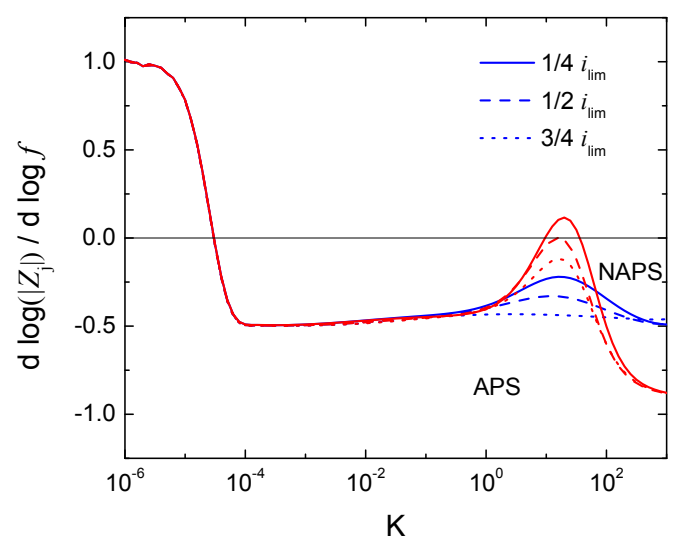

(b)

Figure 10: Calculated derivative of the logarithm of the imaginary part of the global impedance with respect to the logarithm of frequency for the case with a-priori separation (APS) and no a-priori separation (NAPS): a) $0.1 \mathrm{M}$ $\mathrm{AgNO}_{3}$ in a $1 \mathrm{M} \mathrm{KNO}_{3}$ electrolyte; and b) $0.01 \mathrm{M} \mathrm{AgNO}_{3}$ in a $1 \mathrm{M} \mathrm{KNO}_{3}$ electrolyte. 


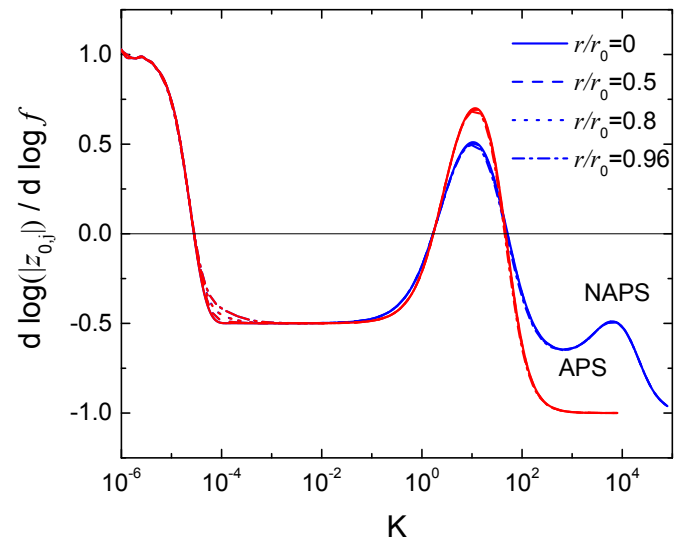

(a)

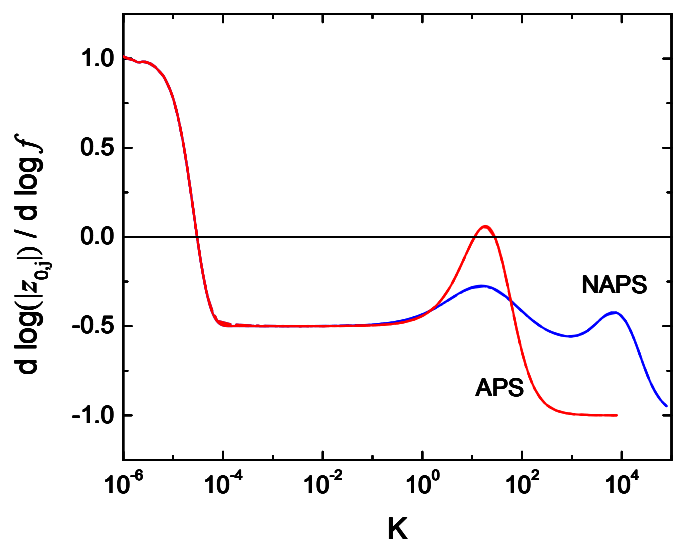

(b)

Figure 11: Calculated derivative of the logarithm of the imaginary part of the local interfacial impedance with respect to the logarithm of frequency for the case with a-priori separation (APS) and no a-priori separation (NAPS): a) $0.1 \mathrm{M} \mathrm{AgNO}_{3}$ in a $1 \mathrm{M} \mathrm{KNO}_{3}$ electrolyte; and b) $0.01 \mathrm{M} \mathrm{AgNO}_{3}$ in a $1 \mathrm{M} \mathrm{KNO}_{3}$ electrolyte. Lines for different radial positions are superposed for the APS and NAPS cases, respectively.

that were described as a pseudo-CPE behavior attributed to the influence of nonuniform current and potential distributions.

The depressed semicircle obtained for the NAPS case corresponds to a high-frequency pseudoCPE behavior with an exponent $\alpha$ between 0.5 and 0.6 , which is much smaller than the values attributed to the influence of nonuniform current and potential distributions.[15] These figures suggest that an additional factor must be contributing to the frequency dispersion when charging and faradaic currents are assumed to be coupled (e.g., NAPS).

\subsection{Local Interfacial Impedance}

The local interfacial impedance is a property of the interface that is not influenced by the ohmic impedance. The derivatives of the logarithm of the local interfacial impedance with respect to the logarithm of frequency corresponding to Figures 8-10 are presented in Figure 11 for APS and NAPS simulations with radial position as a parameter. As expected, the local interfacial impedance is independent of radial position, as are the quantities presented in Figure 11. For the APS case, the derivative at high frequency approaches -1 , indicating that the pseudo-CPE behavior observed in Figure 10 must not be a property of the interface. In contrast, the derivative at high frequency for the NAPS simulations approaches a value larger than -1 , indicating that the pseudo-CPE behavior observed in Figure 10 is a property of the interface and cannot be attributed completely to the influence of nonuniform current and potential distributions. Thus, the coupling of faradaic and charging currents yields a frequency dispersion at frequencies where charging and faradaic processes are both important.

\subsection{Effective Double-Layer Capacitance}

An explanation for the frequency dispersion associated with the coupling of faradaic and charging currents may be obtained by calculation of an effective capacitance that accounts for the influence 


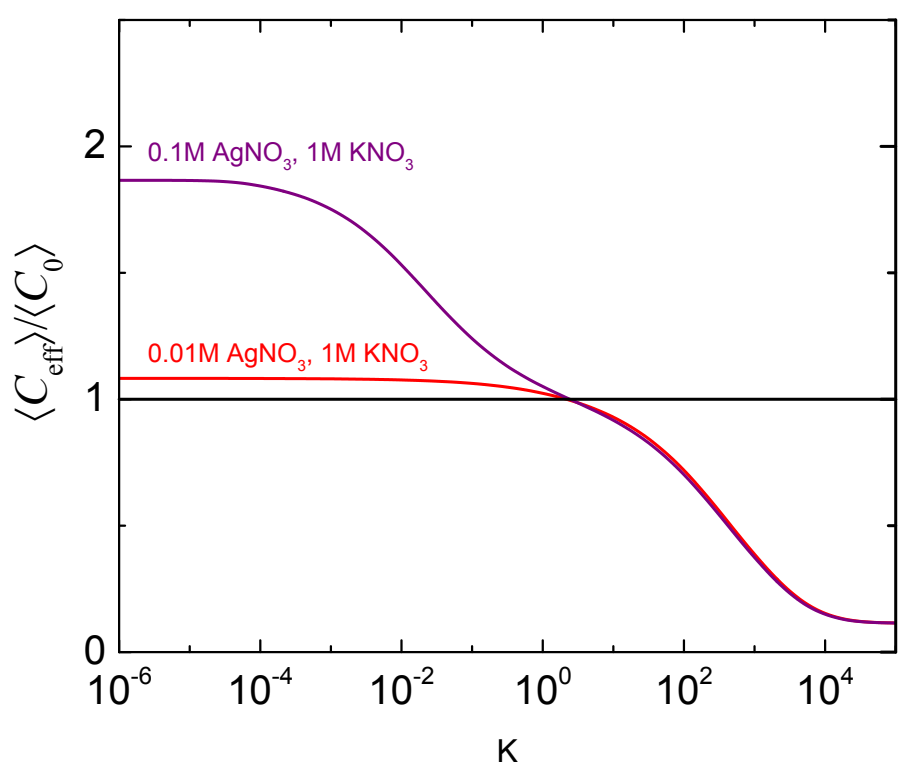

Figure 12: Calculated surface-averaged normalized effective capacitance as a function of dimensionless frequency with electrolyte composition as a parameter.

of potential and concentration on the surface charge. The double-layer capacitance $C_{0}$ is associated with the influence of interfacial potential on electrode charge. The charge on the electrode is also related to the ionic concentration in the solution adjacent to the electrode surface. The effective double-layer capacitance that accounts for the contribution of the flux to the charging process can be defined by

$$
C_{\text {eff }}=C_{0}+\sum_{\mathrm{i}}\left(\frac{\partial \bar{q}_{\mathrm{m}}}{\partial \bar{c}_{\mathrm{i}}(0)}\right)_{\bar{V}, \bar{c}_{\mathrm{j} \neq(\mathrm{i})(0)}} \frac{\widetilde{c}_{\mathrm{i}}(0)}{\widetilde{V}}
$$

The first term on the right-hand side is the double-layer capacitance used in the APS case, and the second term is the contribution of each species in the charging of the double layer.

The calculated surface-averaged normalized effective capacitance is presented in Figure 12 with electrolyte composition as a parameter. At low frequencies, the effective capacitance is independent of frequency. For a large concentration of supporting electrolyte, the effective capacitance at low frequency approaches the capacitance $C_{0}$. At high frequency, however, the effective capacitance is a strong function of frequency, and the curves for large and small concentrations of supporting electrolyte converge. The frequency dependence of $\left\langle C_{\text {eff }}\right\rangle$ at high frequencies is consistent with the frequency dispersion seen in the impedance response.

\section{Discussion}

The simulations of the coupled charging and faradaic currents requires thermodynamic properties of the interface that are not readily available. For the present work, these were obtained under 


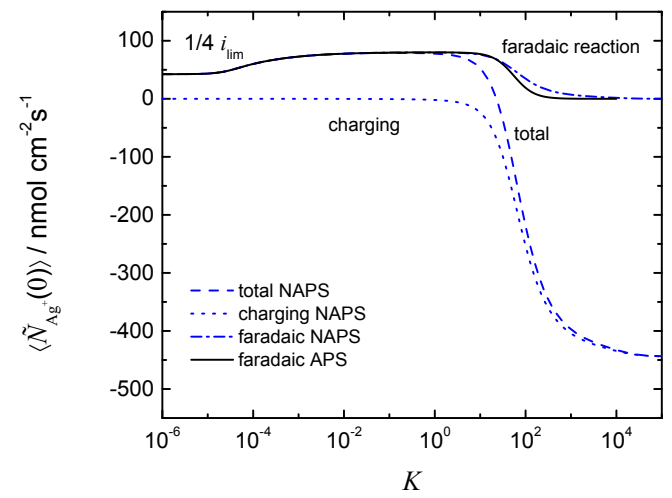

(a)

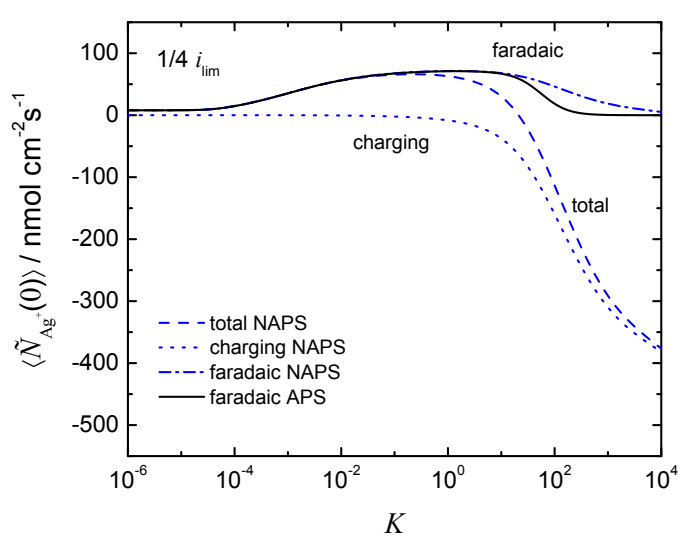

(b)

Figure 13: Calculated contributions to the real part of the oscillating flux defined by equations (31) and (15) as functions of dimensionless frequency: a) $0.1 \mathrm{M} \mathrm{AgNO}_{3}$ in a $1 \mathrm{M} \mathrm{KNO}_{3}$ electrolyte; and b) $0.01 \mathrm{M} \mathrm{AgNO}_{3}$ in a $1 \mathrm{M}$ $\mathrm{KNO}_{3}$ electrolyte.

assumption of a Stern-Gouy-Chapman model of a diffuse double layer in the absence of ion-specific adsorption. Use of a refined model will certainly alter the results.

The work serves to show, however, that the frequency dispersion associated with the coupling of charging and faradaic currents does not disappear in the presence of a large concentration of supporting electrolyte. This result contradicts the conclusions presented by Nisancioglu and Newman.[1] An explanation may be seen by examination of the oscillating component of the calculated fluxes of the reacting species corresponding to Figures 8-12, presented in Figure 13(a) for the case with a small concentration of supporting electrolyte and in Figure 13(b) for the case with a large concentration of supporting electrolyte. The solid black line corresponds to the oscillating flux for the APS case. For the NAPS case, the oscillating part of the flux for silver ions is presented in terms of contributions to the charging and faradaic currents. The charging contribution for the NAPS case is equal to zero at low frequencies and is strongly negative at high frequencies. It is important to note that oscillating term for the NAPS faradaic contribution differs from the corresponding APS term, even for a large supporting electrolyte. Thus, even though a low concentration of silver ions must be associated with a small contribution to the charging current, the contribution to the charging current influences the contribution to the faradaic current and, therefore, influences the impedance response.

The controversy over whether one may decouple faradaic and charging currents that was expressed in the 1960s by papers by Sluyters[2] and Delahay[3] was recently resurrected by the work of Nisancioglu and Newman.[1] The coupling of charging and faradaic currents arises from the contribution of the flux of reacting species to charging the interface as well as to the faradaic reaction. It is evidently appropriate to decouple faradaic and charging currents for systems that are not influenced by mass transfer. The present work suggests, however, that the coupling of faradaic and charging currents should be considered when modeling the impedance response for systems influenced by mass transfer, even for large concentrations of supporting electrolyte. The present work shows that the coupling of faradaic and charging currents results in a frequency dispersion that may appear as a pseudo-CPE behavior. 
Jorcin et al. [17] suggested that CPE behavior, seen in global measurements, can be attributed to time-constants that are distributed either along the electrode surface or in the direction perpendicular (or normal) to the electrode surface. In some cases, the distributions of time constants results in pseudo-CPE behavior in which CPE parameters may appear as functions of frequency. Such was the case for the impedance response reported by Huang et al. [13-15] that was affected nonuniform current and potential distributions. Huang et al. [13] showed that their distributions could be attributed to a surface distribution of time constants. The frequency dispersion associated with coupling of faradaic and charging currents cannot be characterized as being associated with either surface or normal distributions.

\section{Conclusion}

Calculation of the impedance response associated with coupled charging and faradaic currents requires both a detailed microscopic model of the diffuse double layer and solution of coupled convective diffusion equations in the frequency domain. In the present work, a simplified model of the diffuse double layer was used to illustrate the influence on the impedance response of coupling and decoupling the charging and faradaic currents. The coupling of charging and faradaic currents is shown to yield a high-frequency frequency dispersion, demonstrated here for deposition of silver in both poorly-supported and well-supported electrolytes. The frequency dispersion associated with coupled charging and faradaic currents may be explained in terms of an effective capacitance that accounts for the influence of concentration and that is shown to be a function of frequency.

In contrast to reference [1], the coupling of charging and faradaic currents was found to have a significant effect for even well-supported electrolytes. This effect is attributed to the observation that, while the contribution to surface charge by minor species may be small, the separation of reacting species flux to charging and faradaic reactions has a large impact on the faradaic impedance. The associated frequency dispersion is not a $\mathrm{CPE}$, but it may appear as a pseudo-CPE. While the pseudo-CPE caused by nonuniform current and potential distributions was classified as a surface distribution,[13] the frequency dispersion caused by coupled charging and faradaic currents cannot be classified easily as being due to a surface or a normal distribution. 
[1] K. Nisancioglu, J. S. Newman, Separation of double-layer charging and faradaic processes at electrodes, J. Electrochem. Soc. 159 (2012) E59-E61.

[2] J. H. Sluyters, On the impedance of galvanic cells I. theory, Recl. Trav. Chim. Pays-Bas 79 (1960) 1092-1100.

[3] P. Delahay, Electrode processes without a priori separation of double-layer charging, J. Phys. Chem. 70 (7) (1966) 2373-2379.

[4] P. Delahay, G. G. Susbielle, Double-layer impedance of electrodes with charge-transfer reaction, J. Phys. Chem. 70 (10) (1966) 3150-3157.

[5] P. Delahay, K. Holub, G. G. Susbielle, G. Tessari, Double-layer perturbation without equilibrium between concentrations and potential, J. Phys. Chem. 71 (3) (1967) 779-780.

[6] M. Sluyters-Rehbach, Impedances of electrochemical systems: Terminology, nomenclature, and representation: I. cells with metal electrodes and liquid solutions, Pure Appl. Chem. 66 (1994) $1831-1891$.

[7] E. Barsoukov, J. R. Macdonald, Impedance Spectroscopy: Theory, Experiment, and Applications, 2nd Edition, John Wiley \& Sons, 2005.

[8] M. E. Orazem, B. Tribollet, Electrochemical Impedance Spectroscopy, John Wiley \& Sons, Hoboken, NJ, 2008.

[9] S.-L. Wu, Influence of disk electrode geometry on local and global impedance response, Ph.D. thesis, University of Florida, Gainesville, FL (August 2010).

[10] J. S. Newman, K. E. Thomas-Alyea, Electrochemical Systems, 3rd Edition, John Wiley \& Sons, Hoboken, NJ, 2004.

[11] J. O. Bockris, A. K. N. Reddy, Modern electrochemistry: An introduction to an interdisciplinary area, Plenum Press, New York, 1970.

[12] W. G. Cochran, The flow due to a rotating disc, Proceedings of the Cambridge Philosophical Society 30 (1934) 365-375.

[13] V. M.-W. Huang, V. Vivier, M. E. Orazem, N. Pébère, B. Tribollet, The apparent CPE behavior of an ideally polarized disk electrode: A global and local impedance analysis, J. Electrochem. Soc. 154 (2007) C81-C88.

[14] V. M.-W. Huang, V. Vivier, I. Frateur, M. E. Orazem, B. Tribollet, The global and local impedance response of a blocking disk electrode with local CPE behavior, J. Electrochem. Soc. 154 (2007) C89-C98.

[15] V. M.-W. Huang, V. Vivier, M. E. Orazem, N. Pébère, B. Tribollet, The apparent CPE behavior of a disk electrode with faradaic reactions, J. Electrochem. Soc. 154 (2007) C99C107.

[16] M. E. Orazem, N. Pébère, B. Tribollet, Enhanced graphical representation of electrochemical impedance data, J. Electrochem. Soc. 153 (2006) B129-B136.

[17] J.-B. Jorcin, M. E. Orazem, N. Pébère, B. Tribollet, CPE analysis by local electrochemical impedance spectroscopy, Electrochim. Acta 51 (2006) 1473-1479. 


\section{List of Figures}

1 Schematic representation illustrating the contribution of the reacting species to the charging of the electrode-electrolyte interface corresponding to: a) the case with a-priori separation (APS); and b) the case with no a-priori separation (NAPS). . . 2

2 Schematic representation of the electrical double layer structure following a SternGouy-Chapman model. The sketch is not drawn to scale. . . . . . . . . . . . . 5

3 Representation of the different mesh scales used for the finite-element calculations. . 9

4 Calculated capacitance $C_{0}$ for $0.1 \mathrm{M} \mathrm{AgNO}_{3}$ as a function of potential for a silver electrode in solution containing $0.1 \mathrm{M} \mathrm{AgNO}_{3}$ and different concentrations of supporting electrolyte when the system is: a) at equilibrium and b) not at equilibrium.

5 Calculated capacitance $C_{0}$ with fraction of mass-transfer-limiting current as a parameter for $0.1 \mathrm{M} \mathrm{AgNO}_{3}$ as a function of radial position for deposition of silver in an electrolyte containing $0.1 \mathrm{M} \mathrm{AgNO}_{3}$ and different concentrations of supporting electrolyte: a) $1 \mathrm{M} \mathrm{KNO}_{3}$; b) $0.01 \mathrm{M} \mathrm{KNO}_{3} . \ldots \ldots \ldots \ldots \ldots \ldots$

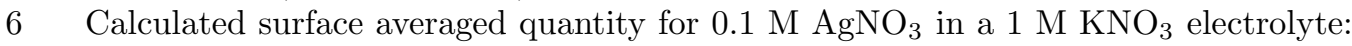

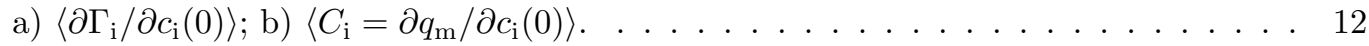

7 Calculated radial distribution of the quantity $C_{\mathrm{i}}=\partial q_{\mathrm{m}} / \partial c_{\mathrm{i}}$ with fraction of the masstransfer-limited current density as a parameter for $0.1 \mathrm{M} \mathrm{AgNO}_{3}$ in a $1 \mathrm{M} \mathrm{KNO}_{3}$ electrolyte: a) $\mathrm{K}^{+}$and $\mathrm{Ag}^{+}$; and b) $\mathrm{NO}_{3}^{-}$. . . . . . . . . . . . . . . . . 12

8 Calculated impedance response in Nyquist format for the case with a-priori separation (APS) and no a-priori separation (NAPS): a) $0.1 \mathrm{M} \mathrm{AgNO}_{3}$ in a $1 \mathrm{M} \mathrm{KNO}_{3}$ electrolyte; and b) $0.01 \mathrm{M} \mathrm{AgNO}_{3}$ in a $1 \mathrm{M} \mathrm{KNO}_{3}$ electrolyte. . . . . . . . . . .

9 Calculated imaginary impedance response as a function of dimensionless frequency for the case with a-priori separation (APS) and no a-priori separation (NAPS): a) $0.1 \mathrm{M} \mathrm{AgNO}_{3}$ in a $1 \mathrm{M} \mathrm{KNO}_{3}$ electrolyte; and b) $0.01 \mathrm{M} \mathrm{AgNO}_{3}$ in a $1 \mathrm{M} \mathrm{KNO}_{3}$ electrolyte. . . . . . . . . . . . . . . . . . . .

10 Calculated derivative of the logarithm of the imaginary part of the global impedance with respect to the logarithm of frequency for the case with a-priori separation (APS)

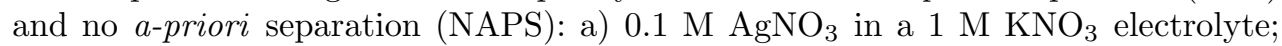
and b) $0.01 \mathrm{M} \mathrm{AgNO}_{3}$ in a $1 \mathrm{M} \mathrm{KNO}_{3}$ electrolyte. . . . . . . . . . . . . . . . . . 14

11 Calculated derivative of the logarithm of the imaginary part of the local interfacial impedance with respect to the logarithm of frequency for the case with a-priori separation (APS) and no a-priori separation (NAPS): a) $0.1 \mathrm{M} \mathrm{AgNO}_{3}$ in a $1 \mathrm{M}$ $\mathrm{KNO}_{3}$ electrolyte; and b) $0.01 \mathrm{M} \mathrm{AgNO}_{3}$ in a $1 \mathrm{M} \mathrm{KNO}_{3}$ electrolyte. Lines for different radial positions are superposed for the APS and NAPS cases, respectively.

12 Calculated surface-averaged normalized effective capacitance as a function of dimensionless frequency with electrolyte composition as a parameter. . . . . . . . . . . 16

13 Calculated contributions to the real part of the oscillating flux defined by equations (31) and (15) as functions of dimensionless frequency: a) $0.1 \mathrm{M} \mathrm{AgNO}_{3}$ in a $1 \mathrm{M}$ $\mathrm{KNO}_{3}$ electrolyte; and b) $0.01 \mathrm{M} \mathrm{AgNO}_{3}$ in a $1 \mathrm{M} \mathrm{KNO}_{3}$ electrolyte. . . . . . . . . 17 